\title{
Barred CO emission in HE 1029-1831*
}

\author{
M. Krips ${ }^{1,2}$, A. Eckart ${ }^{1}$, R. Neri ${ }^{3}$, T. Bertram ${ }^{1}$, C. Straubmeier ${ }^{1}$, S. Fischer ${ }^{1}$, J. G. Staguhn ${ }^{4}$, and S. N. Vogel ${ }^{5}$ \\ 1 I. Physikalisches Institut, Universität zu Köln, Zülpicher Str. 77, 50937 Köln, Germany \\ 2 Harvard-Smithsonian Center for Astrophysics, SMA project, 645 North A'ohoku Place, Hilo, HI 96720, USA \\ e-mail: mkrips@cfa.harvard.edu \\ 3 Institut de Radio Astronomie Millimetrique (IRAM), 300 rue de la Piscine, 38406 Saint Martin d'Hères, France \\ ${ }^{4}$ NASA/Goddard Space Flight Center, Code 665, Building 21, Greenbelt, MD 20771, USA \\ 5 University of Maryland, College Park, MD 20742, USA
}

Received 14 June 2006 / Accepted 29 November 2006

\section{ABSTRACT}

\begin{abstract}
We present $\mathrm{CO}(1-0)$ and $\mathrm{CO}(2-1)$ line emission maps of the barred spiral active galaxy $\mathrm{HE} 1029-1831(z=0.0403)$ obtained with the IRAM Plateau de Bure Interferometer (PdBI) and in part by the Berkeley-Illinois-Maryland Association (BIMA) observatory. The $\mathrm{CO}$ emission is well associated with the optical bar and extended along it. The FWHM of the $\mathrm{CO}$ emission is estimated to be $\sim(6 \pm 2) \mathrm{kpc}$. The $\mathrm{CO}$ emission shows a strong velocity gradient along the minor axis of the bar $\left(\mathrm{PA}=90^{\circ}\right)$. The molecular gas mass is estimated to be $\sim 1.2 \times 10^{10} \mathcal{M}_{\odot}$ which indicates a very gas rich host galaxy. Most of the molecular gas appears to be subthermally excited and cold but we also find weak evidence for a warmer and/or denser gas component at the southern part of the bar emission, about $\sim 4 \mathrm{kpc}$ from the galactic nucleus.
\end{abstract}

Key words. galaxies: active - galaxies: kinematics and dynamics - galaxies: individual: HE 1029-1831 - radio lines: galaxies galaxies: Seyfert

\section{Introduction}

HE 1029-1831 is part of a nearby QSO sample (Bertram et al., in prep.) consisting of $\sim 100$ sources from the Hamburg/ESO survey for bright QSOs. The sole selection criterion of these sources is their low redshift of $z<0.06$ providing the capability of observations on the smallest observationally available angular scales and the accessibility of several important diagnostic lines in the NIR (e.g., the $\mathrm{CO}(2-0)$ rotation vibrational band head absorption line). We aim at determining the distribution and dynamics of molecular gas in the inner $1 \mathrm{kpc}$ of these objects. This will add to a systematic study of the different mechanisms of gas fueling into active galactic nuclei (AGN). Together with the NUGA PdBI survey containing Seyfert and LINER galaxies with $z \leq 0.013$ (García-Burillo et al. 2003, 2003, 2005; Combes et al. 2004; Krips et al. 2005) this study will cover a large part of the activity sequence of AGN. Especially, the role of nuclear embedded bars, rings, spirals as well as mini-spirals, warps and micro-warps in the fueling process is an important issue to investigate possible links between different nuclear disk morphologies and activity types.

Thirty objects of this sample were observed in $\mathrm{CO}$ with the SEST telescope and ten with the BIMA array (Bertram et al., in prep.) resulting in a low detection rate of $\sim 15 \%$ which might be a result of a lack of instrumental sensitivity and uncertainties in the given redshifts; these two observing campaigns were initially conducted to identify the objects with the strongest $\mathrm{CO}$ emission. As a consequence of the low detection rate, we have

* Based on observations carried out with the IRAM Plateau de Bure Interferometer and BIMA. IRAM is supported by INSU/CNRS (France), MPG (Germany) and IGN (Spain). The Berkeley-IllinoisMaryland Association (BIMA) observatory is supported by NSF grant AST 99-81289. carried out deeper integration of an additional 26 sources with the IRAM $30 \mathrm{~m}$ telescope leading to 19 further detections (Betram et al.2006; Bertram et al. in prep.). The BIMA observations resulted in a clear detection of the source HE 1029-1831 and in a tentative one of HE 1136-2304. Both have thus been observed as a follow-up with the IRAM PdBI array but only the first one could be detected and mapped while the tentative BIMA detection of HE 1136-2304 could not be confirmed. HE 11362304 most likely lacks an exact redshift, too.

HE 1029-1831 contains $(m=2)$ spiral arms and a prominent bar $\left(\mathrm{PA} \simeq 30^{\circ}\right)$ in the optical ( $H$-band; Fischer et al. 2006; see Fig. 1). Its redshift was recently determined to be $z=0.0403 \pm 0.0001$ (Kaldare et al. 2003). The classifications of its active nucleus range from an HII region and extreme starburst to an AGN (Kewley et al. 2001). However, recent NIR observations have given evidence that HE 1029-1831 may be classified as a luminous infrared galaxy with a weak narrow line Seyfert nucleus of type 1 (Fischer et al. 2006).

\section{Observations}

\subsection{BIMA}

HE 1029-1831 is one of 10 members of the sample that were scanned for $\mathrm{CO}(1-0)$ emission with BIMA at Hat Creek, CA, USA between October 2000 and April 2001. The interferometer, consisting of 10 antennae with a diameter of $6.1 \mathrm{~m}$ each, was used in $\mathrm{C}$ configuration. The correlator was configured to provide 8 spectral windows in the upper sideband with 32 channels each and covered an overall bandwidth of $800 \mathrm{MHz}$. The observations were scheduled over a range of several months with varying weather conditions, total integration time and uv-coverage for the different sources, hence resulting in different spatial 


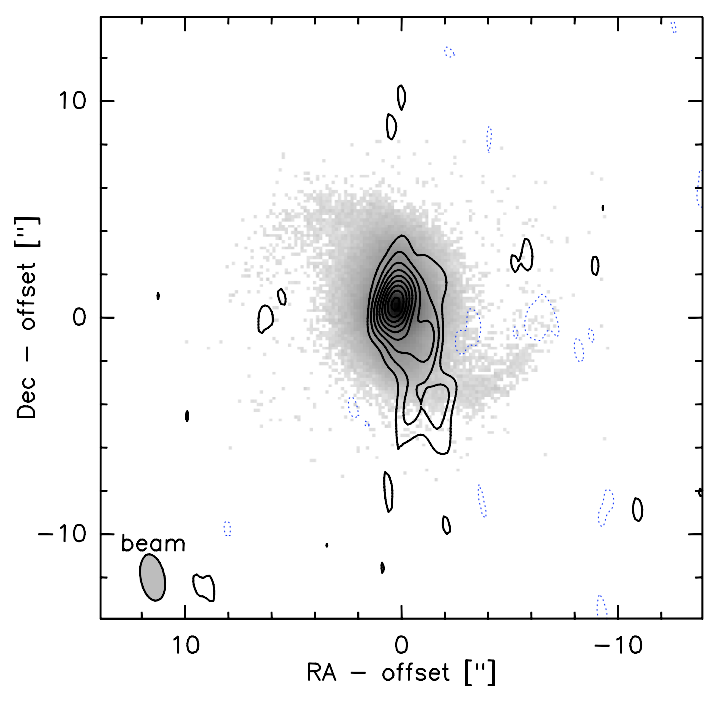

Fig. 1. $H$-band image of HE1029-1831 (from Fischer et al. 2006) superimposed with contours of the integrated $\mathrm{CO}(2-1)$ line emission. Contours are from $3 \sigma=1.4 \mathrm{Jy} /$ beam $\mathrm{km} \mathrm{s}^{-1}$ to $13 \mathrm{Jy} / \mathrm{beam} \mathrm{km} \mathrm{s}^{-1}$ in steps of $3 \sigma$.

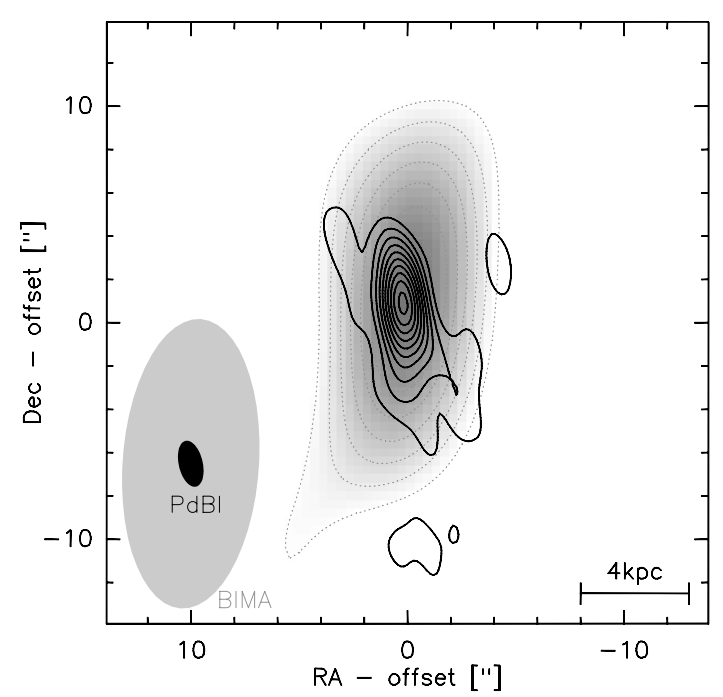

Fig. 2. Integrated $\mathrm{CO}(1-0)$ emission as observed with BIMA (grey scale + dotted grey contours). The PdBI $\mathrm{CO}(1-0)$ (solid black contours) are superimposed on the image. The contour lines for the BIMA data represent multiples of $1 \sigma$ levels, starting at $3 \sigma$, where $1 \sigma=$ $3.0 \mathrm{Jy} /$ beam $\mathrm{km} \mathrm{s}^{-1}$. The contours for the PdBI CO(1-0) emission run from $3 \sigma=0.8 \mathrm{Jy} / \mathrm{beam} \mathrm{km} \mathrm{s}^{-1}$ to $7.3 \mathrm{Jy} /$ beam $\mathrm{km} \mathrm{s}^{-1}$ in steps of $3 \sigma$.

resolutions and detection limits. In the case of HE 1029-1831, the BIMA survey led to a detection of bright $\mathrm{CO}$ line emission (Fig. 2). These data were reduced using 3C273 as bandpass and 1048-191 as gain calibrator. A medium spatial resolution of $13.6^{\prime \prime} \times 5.8^{\prime \prime}$ was achieved in this antenna array configuration.

\section{2. $P d B I$}

As BIMA follow-up, observations of the $\mathrm{CO}(1-0)$ and $\mathrm{CO}(2-1)$ line emission in HE 1029-1831 were conducted in February 2002 with 6 antennae in B and C configurations of the IRAM PdBI and again in March 2003 with 6 antennae in A configuration. The bandpass calibrator was set to $3 \mathrm{C} 273$ and amplitude and phase calibrators were 1048-191 and 1055+018. The latter were observed every twenty minutes. The $3.5 \mathrm{~mm}$ receiver was tuned to the redshifted ${ }^{12} \mathrm{CO}(1-0)$ line and the $1.2 \mathrm{~mm}$ receiver to the redshifted ${ }^{12} \mathrm{CO}(2-1)$ line. The amount of water vapor is calculated to be $\leq 6 \mathrm{~mm}$ for both sets of observations thus providing reasonable weather conditions and system temperatures for a low declination target. At both frequencies we used a total bandwidth of $580 \mathrm{MHz}$ with a frequency resolution of $1.25 \mathrm{MHz}$. The phase tracking centre was set to $\alpha_{2000}=10^{\mathrm{h}} 31^{\mathrm{m}} 57.3^{\mathrm{s}}$ and $\delta_{2000}=-18^{\circ} 46^{\prime} 34.0^{\prime \prime}$ taken from the Nasa/IPAC Extragalactic Database (NED). The total integration time on source (6 antennae) amounted to $\sim 5 \mathrm{~h}$ for the 2002 data and to $\sim 3 \mathrm{~h}$ for the 2003 data.

\section{The data - results}

Both, the $\mathrm{CO}(1-0)$ and $\mathrm{CO}(2-1)$ lines are clearly detected (Figs. 2-4) while only upper limits can be determined for the continuum emission at $3 \mathrm{~mm}$ and $1 \mathrm{~mm}$.

\subsection{CO emission}

$\mathrm{CO}(1-0)$ emission was first detected with the BIMA array (Fig. 2) and confirmed in subsequent observations of $\mathrm{CO}(1-0)$ and $\mathrm{CO}(2-1)$ with the IRAM PdBI. Figure 3 shows the spectrum of the $\mathrm{CO}(1-0)$ and $\mathrm{CO}(2-1)$ line taken at the centroid of the CO emission for the IRAM PdBI and BIMA data. The redshift of the CO emission is determined to be $z=0.0402 \pm 0.0001$ for both transitions and both arrays which is consistent with the optical redshift of $z=0.0403 \pm 0.0001$ (Fig. 3; Kaldare et al. 2003). A gaussian profile has been fitted to both lines. The calculated line parameters are given in Table 1. The integrated maps are plotted in Figs. 2 and 4. Both CO lines are centered at the same position of $\alpha_{\mathrm{J} 2000}=10: 31: 57.3$ and $\delta_{\mathrm{J} 2000}=-18: 46: 33.2$ (Table 1). The CO(1-0) emission is not resolved by the modest angular resolution $\left(\leq 14^{\prime \prime}\right)$ of the BIMA observations; the velocity integrated flux at peak and in the inner $\sim 20^{\prime \prime}$ are almost identical (see Table 1). This excludes the existence of significant, very large scale ( $>10 \mathrm{kpc}$ ) $\mathrm{CO}$ emission. The $\mathrm{CO}$ emission is, however, clearly extended in both transitions at the higher angular resolution $\left(1-3^{\prime \prime}\right)$ of the PdBI and reveals elongations in north to south direction with a position angle (PA) of $\sim 0-20^{\circ}$ (see Table 1) which is slightly smaller than the one derived for the optical bar $\left(\mathrm{PA} \simeq 30^{\circ}\right.$; see Fischer et al. 2006). A combination of the BIMA and IRAM PdBI CO(1-0) maps enables an estimate of the $\mathrm{CO}$ size ( $\equiv F W H M$ ) by fitting an elliptical component to the uv data. We find a FWHM of $\sim(7 \pm 2)^{\prime \prime}$ which corresponds to $\sim(6 \pm 2) \mathrm{kpc}$. The integrated $\mathrm{CO}(1-0)$ line intensity results in a total gas mass of $M\left(\mathrm{H}_{2}+\mathrm{He}\right) \approx 8 \times 10^{9} \mathcal{M}_{\odot}$ for the PdBI map and of $\sim 1.2 \times 10^{10} \mathcal{M}_{\odot}$ for the lower angular resolution BIMA map assuming a $M\left(\mathrm{H}_{2}\right)$ to $L_{\mathrm{CO}}^{\prime}$ conversion factor of $4.8 \mathcal{M}_{\odot}\left(\mathrm{K} \mathrm{km} \mathrm{s}^{-1} \mathrm{pc}^{2}\right)^{-1}$ (Solomon \& Barrett 1991) and $M\left(\mathrm{H}_{2}+\mathrm{He}\right)=1.36 \times M\left(\mathrm{H}_{2}\right)$. The difference in integrated intensity between the PdBI and BIMA maps indicates that the PdBI has filtered out approximately $30 \%$ of the total CO emission due to the lack of short-spacings. Taking the IRAS flux of $3.7 \mathrm{Jy}$ at $3 \mathrm{THz}$ (taken from NED) and assuming optically thin dust emission with a (rest-frame) dust temperature ${ }^{1}$ of $\sim 40 \mathrm{~K}$, we obtain a dust mass of $\sim 1 \times 10^{7} \mathcal{M}_{\odot}$. For a standard galactic gas-to-dust mass ratio of 150 , the expected gas mass would be $\sim 2 \times 10^{9} \mathcal{M}_{\odot}$, i.e., a factor of $\sim 4-6$ lower than using the $\mathrm{CO}$

1 This value was estimated by fitting a grey body spectrum to the IRAS fluxes published by the NED. 
Table 1. Line parameters for $\mathrm{CO}(1-0)$ and $\mathrm{CO}(2-1)$ at the peak and integrated over the central 10-20".

\begin{tabular}{|c|c|c|c|c|c|c|}
\hline Line & $\begin{array}{c}\text { Line } \\
\text { peak flux } \\
\text { [mJy/beam] }\end{array}$ & $\begin{array}{c}\text { Line } \\
\text { width } \\
{\left[\mathrm{km} \mathrm{s}^{-1}\right]}\end{array}$ & $\begin{array}{c}\text { Integrated } \\
\text { peak intensity } \\
{\left[\text { Jy/beam km s }{ }^{-1} \text { ] }\right.}\end{array}$ & $\begin{array}{l}\text { Integrated intensity } \\
\text { in central } \sim 20^{\prime \prime} \\
{\left[\mathrm{Jy} \mathrm{km} \mathrm{s} \mathrm{km}^{-1}\right]}\end{array}$ & $z$ & $\begin{array}{c}M\left(\mathrm{H}_{2}+\mathrm{He}\right)^{b} \\
{\left[10^{9} \mathcal{M}_{\odot}\right]}\end{array}$ \\
\hline \multicolumn{7}{|c|}{$\mathrm{PdBI}$} \\
\hline $\mathrm{CO}(1-0)$ & $65 \pm 7$ & $121 \pm 3$ & $8.4 \pm 0.2$ & $21 \pm 1$ & $0.0402 \pm 0.0001$ & $1-8$ \\
\hline $\mathrm{CO}(2-1)$ & $90 \pm 20$ & $131 \pm 7$ & $12 \pm 0.5$ & $25 \pm 1$ & $0.0402 \pm 0.0001$ & - \\
\hline \multicolumn{7}{|c|}{ BIMA } \\
\hline $\mathrm{CO}(1-0)$ & $190 \pm 30$ & $150 \pm 20$ & $28 \pm 3$ & $30 \pm 3$ & $0.0402 \pm 0.0002$ & $2-12$ \\
\hline & \multicolumn{2}{|c|}{ Centre of $\mathrm{CO}$ emission } & \multicolumn{2}{|c|}{ Dynamical Centre of $\mathrm{CO}$ emission } & $\overline{\mathrm{PA}^{c}}$ & $\overline{\mathrm{i}^{d}}$ \\
\hline & $\mathrm{RA}^{e}$ & $\operatorname{Dec}^{e}$ & $\mathrm{RA}^{e}$ & $\mathrm{Dec}^{e}$ & {$\left[{ }^{\circ}\right]$} & {$\left[{ }^{\circ}\right]$} \\
\hline \multicolumn{7}{|c|}{$\overline{\mathrm{PdBI}}$} \\
\hline $\mathrm{CO}(1-0)$ & $10^{\mathrm{h}} 31^{\mathrm{m}} 57.3^{\mathrm{s}}$ & $-18^{\circ} 46^{\prime} 33.2^{\prime \prime}$ & $10^{\mathrm{h}} 31^{\mathrm{m}} 57.3^{\mathrm{s}}$ & $-18^{\circ} 46^{\prime} 33.4^{\prime \prime}$ & $\sim 20$ & $\sim 10-30$ \\
\hline $\mathrm{CO}(2-1)$ & $10^{\mathrm{h}} 31^{\mathrm{m}} 57.3^{\mathrm{s}}$ & $-18^{\circ} 46^{\prime} 33.2^{\prime \prime}$ & $10^{\mathrm{h}} 31^{\mathrm{m}} 57.3^{\mathrm{s}}$ & $-18^{\circ} 46^{\prime} 33.4^{\prime \prime}$ & $\sim 3$ & - \\
\hline \multicolumn{7}{|c|}{ BIMA } \\
\hline $\mathrm{CO}(1-0)$ & $10^{\mathrm{h}} 31^{\mathrm{m}} 57.3^{\mathrm{s}}$ & $-18^{\circ} 46^{\prime} 33.8^{\prime \prime}$ & $10^{\mathrm{h}} 31^{\mathrm{m}} 57.3^{\mathrm{s}}$ & $-18^{\circ} 46^{\prime} 33.8^{\prime \prime}$ & $\overline{\sim 0}$ & $\sim 10-30$ \\
\hline
\end{tabular}

${ }^{a}$ Taking a luminosity distance of $180 \mathrm{Mpc}$ (based on the following cosmology: $H_{0}=70 \mathrm{~km} \mathrm{~s}^{-1}, \Omega_{m}=0.3$ and $\Omega_{\lambda}=0.7$; $1^{\prime \prime}$ corresponds then to $\sim 0.8 \mathrm{kpc}){ }^{b}$ Assuming a $M\left(\mathrm{H}_{2}\right)$ to $L_{\mathrm{CO}}^{\prime}$ conversion factor of $0.8-4.8 \mathcal{M}_{\odot}\left(\mathrm{K} \mathrm{km} \mathrm{s}^{-1} \mathrm{pc}^{2}\right)^{-1}$ (Solomon \& Barrett 1991; Downes \& Solomon 1998) and $M\left(\mathrm{H}_{2}+\mathrm{He}\right)=1.36 \times M\left(\mathrm{H}_{2}\right) \cdot{ }^{c}$ Estimated position angle of the $\mathrm{CO}$ emission (from North to East). ${ }^{d}$ Estimated inclination angle of the CO disk. ${ }^{e}$ The position uncertainties are of the order of $\sim 0.2^{\prime \prime}$ for the PdBI data and of $\sim 0.4^{\prime \prime}$ for the BIMA data. ${ }^{f}$ Flux errors include $\sim 10 \%(\sim 20 \%)$ uncertainties from the flux calibration at $3 \mathrm{~mm}(1 \mathrm{~mm})$.

luminosity. However, the assumed gas-to-dust mass ratio contains a large uncertainty and can increase in the cases of a few active Ultra-Luminous InfraRed Galaxies (ULIRGs) even up to 1000 or more (e.g., Contini \& Contini 2003) so that the galactic value might not be applicable for HE 1029-1831. Alternatively, Downes \& Solomon (1998) pointed out that the $\mathrm{M}\left(\mathrm{H}_{2}\right)$ to $L_{\mathrm{CO}}^{\prime}$ conversion factor might be lower by a factor of 6 in the circumnuclear regions of ULIRGs.

As the angular resolutions of the PdBI for both transitions are comparable when using uniform weighting for $\mathrm{CO}(1-0)$ and natural weighting for $\mathrm{CO}(2-1)$, we can estimate the central $\left(\leq 8^{\prime \prime}\right)$ line ratio $R_{21}=\mathrm{CO}(2-1) / \mathrm{CO}(1-0)$ (in temperature scale; i.e., $R_{21}=\left(S_{\mathrm{CO} 21}(\mathrm{Jy}) \cdot v_{\mathrm{obs}, \mathrm{CO} 21}^{-2}\right) /\left(S_{\mathrm{CO} 10)}(\mathrm{Jy}) \cdot v_{\mathrm{obs}, \mathrm{CO} 10}^{-2}\right)$ for equal beamsizes) between both lines to be $\simeq 0.5$, indicating subthermal excitation conditions and cold gas. However, one has to keep in mind that this estimate might still be biased by artifacts due to different uv-coverages for both lines in terms of spatial frequencies. One can reduce such a bias by apodising and truncating the respective uv-coverages to the overlapping region. This results in the map of line temperature ratios shown in Fig. 5. The ratio map in this figure is not significantly different from one obtained simply from the unchanged uniformly weighted CO (1-0) and naturally weighted CO (2-1) maps. However, the resulting line ratio map still has to be interpreted with caution as it heavily depends on the adopted weighting factors. Thus, the integrated line ratio must be regarded as a best estimate. Although most of the $\mathrm{CO}$ appears to be subthermally excited and cold, the $\mathrm{CO}(2-1)$ line also indicates asymmetric emission at the south which has only a very weak counterpart in the $\mathrm{CO}(1-0)$ line. Such a difference cannot be explained by resolution effects but could still be accounted for by a residual calibration error in the visibilities and a poor coverage of the uv-plane although no obvious inconsistencies were found. The high line ratio at the southern part of $\sim 1$ might thus indicate different excitation conditions of the gas. The location of the southern CO component is close to the southern spiral arm seen in the optical (see Fig. 1). This might suggest that the $\mathrm{CO}$ at this position traces the contact point of the bar and the southern spiral arm. The high line ratio could be then indicative of gas compression caused by a crossing of the bar- with the spiral arm orbits outside the bar corotation, consistent with
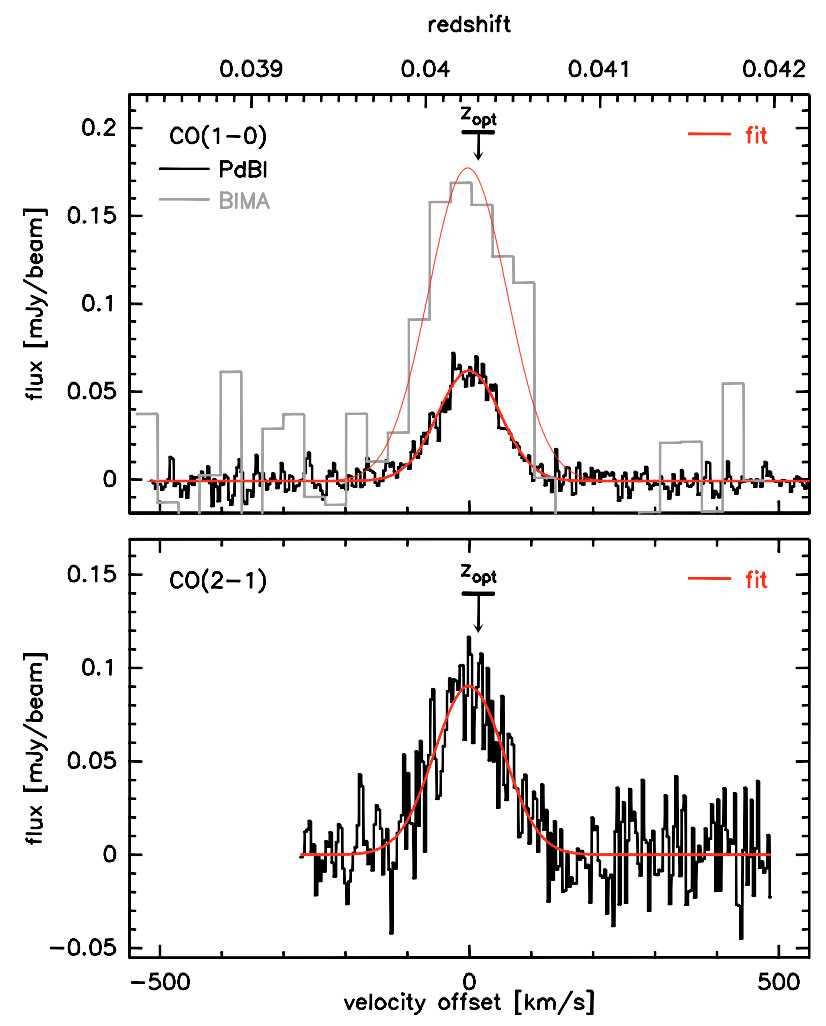

Fig. 3. $\mathrm{CO}(1-0)$ (upper panel) and $\mathrm{CO}(2-1)$ (lower panel) line spectrum taken at the peak emission. The (optical) redshift of $z=0.0403 \pm 0.0001$ is indicated. The velocity resolution is $3.4 \mathrm{~km} \mathrm{~s}^{-1}\left(34 \mathrm{~km} \mathrm{~s}^{-1}\right)$ at both lines for the PdBI (BIMA).

standard theory. However, the southern $\mathrm{CO}$ emission has to be verified by further observations using higher transitions of the $\mathrm{CO}$ line or denser gas tracers such as ${ }^{13} \mathrm{CO}$ or $\mathrm{HCN}$.

We have taken position velocity diagrams at all position angles with a separation of $10^{\circ}$ showing that the cuts in the direction of the $\mathrm{CO}$ extension (position angle: $\mathrm{PA}=0^{\circ}$ ) and perpendicular to it $\left(\mathrm{PA}=90^{\circ}\right)$ represent the extremes in velocity structure: the cuts at $\mathrm{PA}=90^{\circ}$ in both transitions reveal a clear 

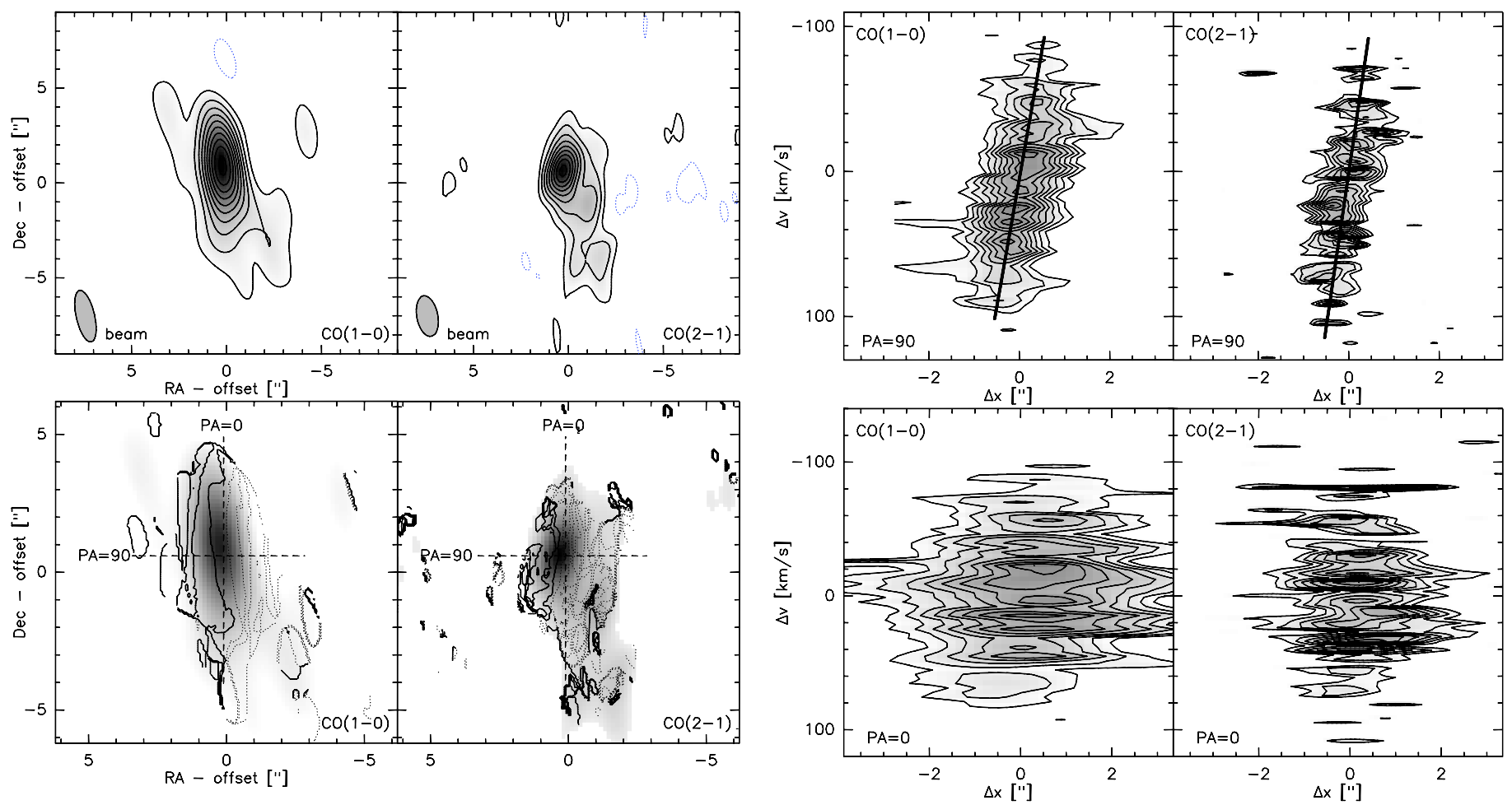

Fig. 4. Upper panel: velocity integrated $\mathrm{CO}(1-0)$ (left) and $\mathrm{CO}(2-1)$ (right) emission in HE 1029-1831 (between $\pm 200 \mathrm{~km} \mathrm{~s}^{-1}$ ). Contours are from $3 \sigma=0.8 \mathrm{Jy} /$ beam km s${ }^{-1}$ ( $1.4 \mathrm{Jy} /$ beam $\mathrm{km} \mathrm{s}^{-1}$ ) to $7.3 \mathrm{Jy} /$ beam $\mathrm{km} \mathrm{s}^{-1}\left(13 \mathrm{Jy} /\right.$ beam km s$\left.{ }^{-1}\right)$ in steps of $3 \sigma$ for $\mathrm{CO}(1-0)(\mathrm{CO}(2-1))$. Lower panel: iso-velocity maps of the $\mathrm{CO}(1-0)$ and $\mathrm{CO}(2-1)$ emission $\mathrm{CO}(1-0)$ (left) and $\mathrm{CO}(2-1)$ (right) emission in HE 1029-1831. Contours are around the dynamic centre in steps of $10 \mathrm{~km} \mathrm{~s}^{-1}$. The dashed lines indicate the cuts along which the position-velocity diagrams in Fig. 6 were taken.

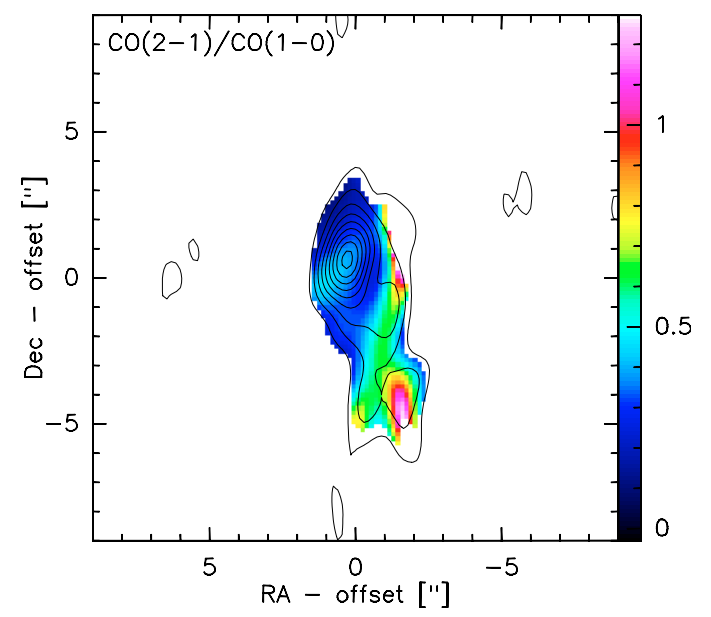

Fig. 5. The $\mathrm{CO}(2-1) / \mathrm{CO}(1-0)$ line ratio in temperature scale (color scale) overlaid with the velocity integrated $\mathrm{CO}(2-1)$ emission (contours). Increments for the velocity integrated $\mathrm{CO}(2-1)$ emission are the same as in Fig. 4.

and strong velocity gradient (Fig. 6, upper panels) which extends over a positional range of $\pm 0.5^{\prime \prime}$; by comparison, velocity gradients along the $\mathrm{CO}$ elongation, i.e., at $\mathrm{PA}=0^{\circ}$, are weaker and much less apparent (Fig. 6, lower panels). Assuming a radius of $\sim 1^{\prime \prime}$ equivalent to $0.8 \mathrm{kpc}$ and a velocity of $\sim 80 \mathrm{~km} \mathrm{~s}^{-1}$, the total dynamical mass within the central $0.8 \mathrm{kpc}$ - not corrected for inclination effects - can be calculated to be $\geq 2 \times 10^{9} \mathcal{M}_{\odot}$. Probably,

Fig. 6. The upper panels show the position velocity diagrams taken across the bar $\left(\mathrm{PA}=90^{\circ}\right)$, and the lower panel those of a cut perpendicular to it, i.e., along the bar ( $\mathrm{PA}=0^{\circ}$; see Fig. 4). Increments are in steps of $10 \%$ from $20 \%$ and $30 \%$ of the maximum for the $\mathrm{CO}(1-0)$ and the $\mathrm{CO}(2-1)$ line respectively. The thick lines indicate the steepness of the gradient at $\mathrm{PA}=90^{\circ}$ and are supposed to guide the reader's eyes.

the gas disk is seen closer to face-on than to edge-on (compare also Fischer et al. 2006) so that this value must be regarded as lower limit. Since the dynamical mass includes the mass of the gas and of the stars, we can estimate the inclination of the galaxy by assuming that the gas mass of $\sim 0.2-1.2 \times 10^{10} \mathcal{M}_{\odot}$ contributes $10 \%$ to the total mass of the galaxy. This results in an inclination of $\sim 5-10^{\circ}$. By equating directly the estimated gas mass with the dynamical mass, the inclination would increase to $<15-30^{\circ}$ giving a more conservative upper limit.

\subsection{Continuum emission}

The continuum emission at $3 \mathrm{~mm}$ and $1 \mathrm{~mm}$ was not detected. The upper limits were determined in the line free channels (i.e., at velocities lower than $-250 \mathrm{~km} \mathrm{~s}^{-1}$ and larger than $+250 \mathrm{~km} \mathrm{~s}^{-1}$ at $3 \mathrm{~mm}$ and at velocities lower than $-200 \mathrm{~km} \mathrm{~s}^{-1}$ and larger than $-200 \mathrm{~km} \mathrm{~s}^{-1}$ at $1 \mathrm{~mm}$ ) to $3 \sigma=3 \mathrm{mJy}$ at $3 \mathrm{~mm}$ and to $3 \sigma=8 \mathrm{mJy}$ at $1 \mathrm{~mm}$. The two upper limits are compatible with the $\mathrm{mm}$ values predicted by the grey body spectrum of the (infrared) dust emission (compare previous section) which are of the order of 1-5 mJy.

\section{Gas dynamics}

The kinematics and distribution of the CO emission in HE 10291831 suggest a barred potential in this galaxy (compare Roberts et al. 1979) as visible in the optical (Fischer et al. 2006). We have simulated the gas emission in HE 1029-1831 by adopting the bar approach from Schinnerer et al. (2000) and Telesco \& Decher (1988). Instead of computing the gas motions in a given gravitational potential, the gas is simply split up into several closed 

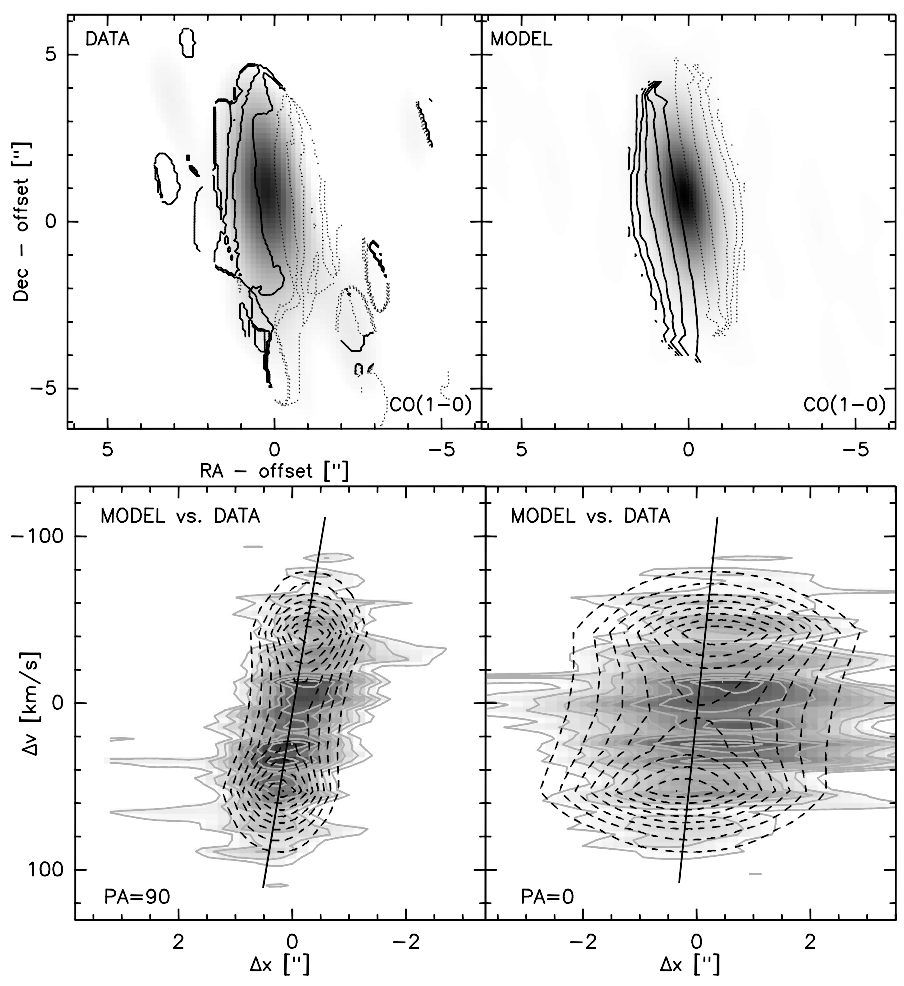

Fig. 7. Upper panel: iso-velocity diagrams of the $\mathrm{CO}(1-0)$ emission in HE 1029-1831 (left) and of a first-order bar model (right) overlaid to the respective integrated line emission maps. Increments are again in steps of $10 \mathrm{~km} \mathrm{~s}^{-1}$. Lower panel: position-velocity diagram simulated with a bar model for the emission in HE 1029-1831 taken along a cut with a PA of $90^{\circ}$ (left) and of $0^{\circ}$ (right); compare Figs. 4 and 6. The dashed contours are superimposed on the observed data presented in Fig. 6. Increments are in steps of $10 \%$ from $20 \%$ of the maximum.

orbits with continous, non-overlapping curves of ellipticities $\epsilon(r)$ and position angles $(\mathrm{pa}(r))$ which are both smooth functions of the radius. Each ring, i.e., each radius has also an assigned velocity $^{2}$. For simplification, we adopt a constant rotation curve and a constant intensity over all rings. As fixed input parameters, we used the estimated PA of the emission of $\sim 0-20^{\circ}$ and the inclination of $\sim 10-30^{\circ}$. To find the best-fit model, we compared the simulated distribution and kinematics for different sets of $\epsilon(r)$ and $\mathrm{pa}(r)$ with those from the observed gas emission. Figure 7 shows the result of these simulations. To facilitate a comparison to the observed $\mathrm{CO}(1-0)$ data, the simulated $\mathrm{CO}$ distribution has been transformed to a uv-table based on the uv-coverage from the $\mathrm{CO}(1-0) \mathrm{PdBI}$ observations; this is a more accurate method than just convolving the model with the beamsize of the $\mathrm{CO}(1-$ 0 ) observations as it considers also spatial filtering effects due to the discrete uv-coverage. This simplified model already reproduces the observed emission. The simulated distribution and the kinematics of the gas agree very well with each other indicating that the gas properties can be almost completely explained by assuming a barred potential. However, the asymmetry between the $\mathrm{CO}(1-0)$ and $\mathrm{CO}(2-1)$ line cannot be explained by the symmetric bar model.

2 The used code 3DRings is described in more detail by Schinnerer et al. (2000).

\section{Summary and conclusions}

Strong and extended $\mathrm{CO}(1-0)$ and $\mathrm{CO}(2-1)$ emission is detected in the barred Seyfert galaxy HE 1029-1831 with the IRAM PdBI and the BIMA array while no continuum emission was found. Both lines agree well with each other in distribution, position and velocity width and imply a total gas mass of $\sim 1.2 \times 10^{10} \mathcal{M}_{\odot}$. The difference between the dynamical mass $\left(\sim 2 \times 10^{9} \mathcal{M}_{\odot}\right.$, not corrected for inclination) indicates a low inclination of $\sim 10$ $30^{\circ}$. The $\mathrm{CO}(1-0)$ and $\mathrm{CO}(2-1)$ emission are extended along the optical bar. The FWHM of the $\mathrm{CO}$ emission is calculated to be $\sim(6 \pm 2) \mathrm{kpc}$. Also, a striking velocity gradient is found at a position angle of $90^{\circ}$, i.e., along the minor axis of the bar, extending over $\sim 1^{\prime \prime}$ approximately. In the direction of the bar $\left(\mathrm{PA}=0^{\circ}\right)$, almost no velocity gradient is seen. Adopting the approach from Schinnerer et al. (2000), the line emission can be simulated with a very simple bar model explaining the detected distribution and kinematics. Following Roberts et al. (1979), the strong velocity gradient across the bar $\left(\mathrm{PA}=90^{\circ}\right)$ can be interpreted as bar-driven inflow of the gas while the motions along the bar $\left(\mathrm{PA}=0^{\circ}\right)$ indicate a velocity dispersion of $\Delta v= \pm 80 \mathrm{~km} \mathrm{~s}^{-1}$. The line ratio between $\mathrm{CO}(2-1)$ and $\mathrm{CO}(1-0)$ is estimated to be $\sim 0.5$ over most areas of the bar although probably still biased by resolution effects. This indicates subthermally excited and cold gas which is typical for gas in a bar/disk. The southern increase of the $\mathrm{CO}(2-1) / \mathrm{CO}(1-0)$ line ratio to over 1 indicates different excitation conditions which might be caused by gas compression at the crossing point of the bar and the southern spiral arm. However, this southern $\mathrm{CO}$ component still requires further support.

Acknowledgements. Part of this work was supported by the German Sonderforschungsbereich, SFB, project number 494. MK was partly funded by a predoctoral fellowship of the german academic exchange service (DAAD) and the french government. This research has made use of the NASA/IPAC Extragalactic Database (NED) which is operated by the Jet Propulsion Laboratory, California Institute of Technology, under contract with the National Aeronautics and Space Administration. We thank the anonymous referee for useful and careful comments.

\section{References}

Bertram, T., Eckart, A., Krips, M., et al. 2006, New Astron. Rev., 50, 712

Combes, F. 2000, Dynamics of Galaxies: from the Early Universe to the Present, 15th IAP meeting held in Paris, France, July 9-13, 1999, ed. F. Combes, G. A. Mamon, \& V. Charmandaris, ASP Conf. Ser., 197, 15

Combes, F. 2004, The Interplay among Black Holes, Stars and ISM in Galactic Nuclei, ed. T. Storchi-Bergmann, L. C. Ho, \& Henrique R. Schmitt., (Cambridge, UK: Cambridge University Press), Proc. IAU Symp., 222, 383 Combes, F., García-Burillo, S., Boone, F. et al. 2004, A\&A, 414, 857

Contini, M., \& Contini, T. 2003, MNRAS, 342, 299

Downes, D., \& Solomon, P. M. 1998, ApJ, 507, 615

Fischer, S., Iserlohe, C., Zuther, J., et al. 2006, A\&A, 452, 827

García-Burillo, S., Combes, F., Eckart, A., et al. 2003a, in Active Galactic Nuclei: From Central Engine to Host Galaxy, ed. S. Collin, F. Combes \& I. Shlohsman, ASP Conf. Ser., 290, 423

García-Burillo, S., Combes, F., Hunt, L. K., et al. 2003b, A\&A, 407, 485

García-Burillo, S., Combes, F., Schinnerer, E., Boone, F., \& Hunt, L. K. 2005, A\&A, 441, 1011

Kaldare, R., Colless, M., Raychaudhury, S., \& Peterson, B. A. 2003, MNRAS, 339,652

Kewley, L. J., Heisler, C. A., Dopita, M. A. \& Lumsden, S. 2001, ApJS, 132, 37 Krips, M., Eckart, A., Neri, R., et al. 2005, A\&A, 442, 479

Roberts, W. W., Huntley, J. M., \& van Albada, G. D. 1979, ApJ, 233, 67

Schinnerer, E., Eckart, A., \& Tacconi, L. J. 2000, ApJ, 533, 826

Solomon, P. M., \& Barrett, J. W. 1991, Dynamics of Galaxies and Their Molecular Cloud Distributions, Proceedings of the 146th Symposium of the International Astronomical Union, held in Paris, France, June 4-9, 1990, ed. F. Combes and F. Casoli, International Astronomical Union, Symposium , (Dordrecht: Kluwer Academic Publishers), 146, 235

Telesco, C. M., \& Decher, R. 1988, ApJ, 334, 573 